\title{
Cardiovascular highlights from non-cardiology journals
}

\author{
Steven M Bradley, JournalScan Editor
}

\section{BETA-BLOCKERS IN HEART FAILURE - WHAT IS THE IMPACT OF CONCURRENT ATRIAL FIBRILLATION?}

Beta-blockers form an essential cornerstone of therapy for heart failure with reduced ejection fraction. However, the benefits of beta-blockade for heart failure in patients with concurrent atrial fibrillation are less certain. In this meta-analysis of individualpatient data from 10 randomized control trials comparing betablockers to placebo for systolic heart failure, investigators assessed the impact of beta-blockade by the presence of sinus rhythm or atrial fibrillation at baseline. Of the 18,254 patients assessed, $76 \%$ were in sinus rhythm and $17 \%$ were in atrial fibrillation at baseline. Over 1.5 years of mean follow-up, betablocker therapy was associated with a significant mortality benefit in sinus rhythm patients (HR 0.73, 0.67-0.80; $\mathrm{p}<0.001$ ) but not in patients with atrial fibrillation (HR 0.97, $0.83-1.14 ; \mathrm{p}=0.73$ ). Results did not vary by subgroup of patients with atrial fibrillation or for secondary outcomes which included cardiovascular death, cardiovascular hospital admission, and non-fatal stroke.

\section{Conclusion}

The benefits of beta-blocker treatment in heart failure appear reduced by concomitant atrial fibrillation. Although the authors suggest $\beta$ blockers should not be used preferentially for rate control in patients with atrial fibrillation and systolic heart failure, it is important to note this was not a comparative effectiveness study and there is insufficient data to support this conclusion.

Summarized by Steven M Bradley and Jehu S Mathew

- Kotecha D, Holmes J, Krum H, et al.; on behalf of the Beta-Blockers in Heart Failure Collaborative $\mathrm{G}$. Efficacy of beta blockers in patients with heart failure plus atrial fibrillation: An individual-patient data meta-analysis. Lancet 2014. pii: S0140-6736(14) 61373-8. doi: 10.1016/50140-6736(14)61373-8.

\section{QUALITY OF LIFE FOLLOWING CORONARY ARTERY BYPASS GRAFT SURGERY VERSUS GUIDELINE-BASED MEDICAL THERAPY IN ISCHEMIC CARDIOMYOPATHY}

It remains uncertain if surgical revascularization improves patient outcomes in the setting of coronary artery disease with reduced systolic function. This is particularly true in light of the STICH trial, a contemporary randomized control trial of coronary artery bypass graft surgery (CABG) with optimal medical therapy compared to optimal medical therapy alone in patients with ischemic left ventricular dysfunction. The primary results of the STICH trial demonstrated no overall mortality benefit to CABG with medical therapy compared to medical therapy alone. In this study, the trial authors report quality of life outcomes from the STICH trial. The trial randomized 1212 patients with an ejection fraction $\leq 35 \%$ with coronary anatomy suitable for surgical revascularization to either CABG with medical therapy or medical therapy alone. Quality of life assessments were performed at 4, 12, 24, and 36 months using validated patient-reported outcome measures, including the Kansas City Cardiomyopathy Questionnaire (KCCQ), the Seattle Angina Questionnaire (SAQ), Center for Epidemiologic Studies Depression Scale, Cardiac Self-Efficacy Questionnaire, and EuroQol-5D. Patients receiving CABG with medical therapy had better quality of life scores in follow-up. Given the challenges of interpreting the clinical importance of these quality of life scores, the authors also reported the proportion of patients who achieved clinically meaningful improvements in KCCQ scores. This analysis demonstrated a number-needed-to-treat with CABG of between 9 and 14 patients to achieve one additional patient with a clinically meaningful improvement in KCCQ score.

\section{Conclusions}

Although the CABG for ischemic cardiomyopathy did not reduce all-cause mortality in the STICH trial, health-related quality of life measures consistently favored CABG with medical therapy over medical therapy alone through 36 months of follow-up.

Summarized by Javier A Valle and Steven M Bradley

- Mark DB, Knight JD, Velazquez EJ, et al. Quality-of-Life Outcomes With Coronary Artery Bypass Graft Surgery in Ischemic Left Ventricular Dysfunction: A Randomized Trial. Ann Intern Med 2014;161:392-9.

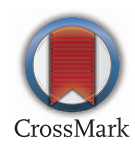

To cite Bradley SM. Heart 2015;101:82. 